\title{
Conhecimento, atitudes e comportamentos frente ao risco ocupacional de exposição ao HIV entre estudantes de Medicina da Faculdade de Medicina da Universidade Federal de Minas Gerais
}

\author{
Knowledge, attitudes and behavior in relation to occupational \\ risk for HIV among medical students of the School of \\ Medicine Universidade Federal de Minas Gerais \\ Antonio Carlos C. Toledo Júnior, Fávia Andrade Ribeiro, Flávia Gomes Faleiro \\ Ferreira, Rodrigo Moraes Ferraz e Dirceu Bartolomeu Greco
}

\begin{abstract}
Resumo Os objetivos deste trabalho são avaliar risco ocupacional de exposição ao HIV entre estudantes de medicina, avaliar o conhecimento e o uso das medidas de biossegurança e a cobertura da vacinação contra hepatite $B$. Questionário específico foi aplicado a 136 estudantes de medicina da Universidade Federal de Minas Gerais. A maioria da população avaliada, 87 (64\%) estudantes relataram participar de procedimentos cirúrgicos, 68 (50\%) já sofreram algum tipo de exposição a sangue, 90 (66,2\%) afirmaram conhecer as medidas universais de biossegurança e $33(24,3 \%)$ relataram conhecer os procedimentos em caso de exposição a sangue contaminado. A população avaliada apresenta alto risco de exposição a material biológico e cerca de um terço desta tem baixo nível de conhecimento das medidas de biossegurança, apesar de fazer uso delas. Torna-se necessário a utilização de estratégias como ensino formal, treinamento em serviço e criação de serviços especializados em biossegurança para a modificação desta realidade.
\end{abstract}

Palavras-chaves: Infecção pelo HIV. AIDS. Risco ocupacional. Prevenção.

Abstract The objectives of this study were to evaluate the occupational risk of medical students, their knowledge and practice of universal biosafety measures and hepatitis $B$ immunization coverage. A specific questionnaire was applied to 136 medical students of the Universidade Federal de Minas Gerais: 87 (64\%) students were involved in surgical procedures, $68(50 \%)$ had been exposed to blood, 90 (66.2\%) knew the universal biosafety measures and 33 (24.3\%) knew the procedure in case of blood exposure. Thus, this population has a high risk of blood exposure and although there is a low formal knowledge about universal biosafety measures, most of these were generally used. New strategies, such as formal teaching of universal biosafety measures, in loco supervision, and biosafety teams, are necessary to change this situation.

Key-words: HIV infection. AIDS. Occupational risk. Prevention.

\footnotetext{
Serviço de Doenças Infecciosas e Parasitárias, Departamento de Clínica Médica da Faculdade de Medicina da Universidade Federal de Minas Gerais, Belo Horizonte, MG.

Apoio financeiro: Pró-Reitoria de Pesquisa da Universidade Federal de Minas Gerais.

Endereço para correspondência: Dr. Antonio Carlos C. Toledo Júnior. R. Mário Coutinho 156/304, 30570-310 Belo Horizonte, MG, Brasil. Tel: 5531 273-5626; Fax: 5531 273-0422.

E-mail: toledoac@task.com.br

Recebido para publicação em 1/9/98.
} 
A epidemia do HIV/AIDS foi identificada no início dos anos 80, quando pesquisadores dos Centers for Disease Control and Prevention norteamericano (CDC) observaram a ocorrência de sarcoma de Kaposi e pneumonia por Pneumocystis carinii em jovens homossexuais previamente hígidos $^{6813}$. Desde então esta epidemia encontrase em franca expansão. Em 1998, estima-se que 16.000 pessoas se infectam pelo HIV a cada dia, a maioria em países em desenvolvimento, principalmente na África, sul da Ásia e América Latina (UNAIDS, Genebra 1998). A Organização Mundial de Saúde (OMS) estima que, até o final de 1997, ocorreram 29,4 milhões de casos de infecção pelo HIV, 8,4 milhões de casos de AIDS e 6,4 milhões de mortes. A Organização Panamericana de Saúde (OPAS) registrou 785.422 casos de AIDS nas Américas até março de 1997, com 461.421 mortes. No Brasil, foram notificados 128.821 casos de AIDS até fevereiro de 1997, sendo 8.221 casos em Minas Gerais e 2.776 em Belo Horizonte ${ }^{9}$.

A transmissão do HIV ocorre principalmente através da exposição a material biológico que contenha o HIV livre e/ou células infectadas pelo vírus. O sangue e seus derivados, o sêmen, as secreções vaginais são os principais veículos de transmissão². O suor, a lágrima, a urina e as fezes, sem contaminação grosseira pelo sangue, não são considerados materiais infectantes. A transmissão por saliva é extremamente rara, ocorrendo apenas em situações de contato íntimo, logo ela também não é considerada material infectante. O leite materno, que só é considerado como veículo de transmissão através do aleitamento, também não é considerado material infectante em outras situações ${ }^{4}$.

Os profissionais de saúde, envolvidos na assistência direta a pacientes, ou aqueles que manipulam ou têm contato com materiais biológicos potencialmente contaminados, apresentam risco não só à infecção pelo HIV, mas também a outros agentes infecciosos, como os vírus das hepatites B e C, Trypanossoma cruzi e Treponema pallidum ${ }^{12}$.

Do início da epidemia do HIV/AIDS até junho de 1997, o CDC registrou 52 casos comprovados de infecção pelo HIV entre profissionais da área de saúde nos Estados Unidos. Outros 114 casos prováveis foram registrados no mesmo período. Os acidentes péfuro-cortantes com agulhas são o tipo de exposição mais freqüente ${ }^{3}$. O risco de soroconversão após um acidente percutâneo com sangue sabidamente contaminado é de 0,3\% (IC95\% - 0,2\% a 0,5\%) e após exposição de mucosa é de 0,09\% (IC95\% - 0,006\% a 0,5\%) ${ }^{1}$ O risco de infecção varia de acordo com a carga viral do paciente, o tipo (sólida ou oca) e calibre da agulha, utilização ou não de luvas e volume de sangue inoculado.

A prevenção deste tipo de exposição é feita, principalmente através das medidas universais de biossegurança (MUB) em situações potenciais de risco. Mesmo com a utilização das MUB, situações de emergência que envolvem risco de vida do paciente e aquelas relacionadas a ensino e treinamento envolvem maior risco de acidentes. $O$ avanço do tratamento anti-retroviral, com drogas potentes e com diferentes mecanismos de ação, possibilitou a definição de esquemas terapêuticos pós-exposição (Tabela 1) ${ }^{4}$.

Apesar do conhecimento do risco de infecção pelo HIV e por outros agentes, as atitudes dos profissionais de saúde geralmente são negligentes em relação as MUB.

Os objetivos deste trabalho são: 1) estimar risco ocupacional de exposição ao HIV entre estudantes de medicina; 2) identificar o grau de conhecimento sobre as medidas de biossegurança entre estudantes de medicina; 3) avaliar o uso de medidas de biossegurança entre estudantes de medicina; 4) identificar a cobertura vacinal contra hepatite $B$.

\section{MATERIAL E MÉTODOS}

Questionário. Foi desenvolvido questionário fechado, de autopreechimento, específico para este estudo. O preenchimento foi voluntário e o questionário era anônimo e não vinculado. Foram realizadas as seguintes perguntas: idade; sexo; período atual do curso; "realiza rotineiramente algum procedimento cirúrgico, como suturas, partos ou cirurgias?; faz uso de óculos de proteção?; faz uso de máscara cirúrgica?; faz uso de óculos de luvas?; faz uso de avental cirúrgico?; o material de proteção é seu?; você conhece as medidas de biossegurança?; você faz uso delas?; você acha que está exposto a infecção pelo VHB ou HIV durante o curso de medicina?; você se lembra de alguma matéria que ensinou medidas de biossegurança formalmente?; você é vacinado contra hepatite $B$ ?; você já sofreu algum acidente pérfurocortante? alguma vez já espirrou sangue em seus 
Tabela 1 - Recomendações para quimioprofilaxia após a exposição ocupacional ao HIV10.

\begin{tabular}{|c|c|c|c|}
\hline Tipo de exposição & Material fonte & Profilaxia $^{1}$ & Esquema anti-retroviral $^{2}$ \\
\hline \multirow[t]{6}{*}{ Percutânea } & a) Sangue ${ }^{3}$ & & \\
\hline & - risco mais elevado & Recomendar & $\mathrm{AZT}+3 \mathrm{TC}+\mathrm{IP}^{4}$ \\
\hline & - risco aumentado & Recomendar & $\mathrm{AZT}+3 \mathrm{TC}+\mathrm{IP}^{4}$ \\
\hline & - sem risco aumentado & Oferecer & $\mathrm{AZT}+3 \mathrm{TC}$ \\
\hline & $\begin{array}{l}\text { b) Líquido orgânico: contendo sangue visível, } \\
\text { outro líquido ou tecido potencialmente } \\
\text { infeccioso }\end{array}$ & Oferecer & $\mathrm{AZT}+3 \mathrm{TC}$ \\
\hline & c) Outro líquido corporal (p.ex. urina) & Não oferecer & \\
\hline \multirow[t]{3}{*}{ De mucosa } & a) Sangue & Oferecer & $\mathrm{AZT}+3 \mathrm{TC}+\mathrm{IP}^{4}$ \\
\hline & $\begin{array}{l}\text { b) Líquido orgânico contendo sangue visível, } \\
\text { outro líquido ou tecido potencialmente infeccioso }{ }^{5}\end{array}$ & Oferecer & $\mathrm{AZT}+3 \mathrm{TC}$ \\
\hline & c) Outro líquido corporal (p.ex. urina) & Não oferecer & \\
\hline \multirow[t]{3}{*}{ De pele, risco aumentado 6} & a) Sangue & Oferecer & $\mathrm{AZT}+3 \mathrm{TC}+\mathrm{IP}^{4}$ \\
\hline & $\begin{array}{l}\text { b) Líquido orgânico contendo sangue visível, } \\
\text { outro líquido ou tecido potencialmente infeccioso }{ }^{5}\end{array}$ & Oferecer & $\mathrm{AZT}+3 \mathrm{TC}$ \\
\hline & c) Outro líquido corporal (p.ex. urina) & Não oferecer & \\
\hline
\end{tabular}

${ }^{1}$ Recomendar: a profilaxia pós-exposição deve ser recomendada ao profissional exposto, com aconselhamento. Oferecer: a profilaxia pós-exposição deve ser oferecida ao profissional exposto, com aconselhamento. Não oferecer: a profilaxia pós-exposição não deve ser oferecida, pois não houve exposição ocupacional ao HIV.

2 Esquema Anti-retroviral: AZT 200mg três vezes ao dia; 3TC 150mg duas vezes ao dia; indinavir $800 \mathrm{mg}$ três vezes ao dia por 4 semanas. A opção pelo indinavir deve-se à sua melhor tolerância. Na falta ou impossibilidade do seu uso, deve-se preferir o ritonavir (600mg duas vezes ao dia, por 4 semanas) ao saquinavir, devido à baixa biodisponibilidade deste último.

${ }^{3}$ Risco Mais Elevado: presença de ambos: maior volume de sangue (por exemplo, ferimento por agulha de grosso calibre) e sangue contendo alto teor de HIV (por exemplo, doença retroviral aguda ou AIDS terminal). Risco Aumentado: presença de um dos dois: maior volume de sangue ou sangue contendo alto teor de HIV. Sem Risco Aumentado: ausência de ambos os fatores de risco.

${ }^{4} \mathrm{IP}$ = inibidor de protease, com opção pelo indinavir devido ao fato de ser melhor tolerado. Na impossibilidade do seu uso, recomendase ritonavir.

${ }^{5}$ Inclui: sêmen, secreção vaginal, líquor, líquido sinovial, peritoneal, pericárdico e amniótico.

${ }^{6}$ Para a pele, o risco é maior para exposição que envolva um alta carga de HIV, contato prolongado, área extensa, ou uma área na qual a integridade da pele está visivelmente comprometida; para exposição de pele sem maior risco, o risco de toxicidade ultrapassa o benefício da profilaxia pós-exposição.

Observações:

a) em situações em que a condição sorológica do paciente-fonte não for conhecida deve-se:

- iniciar esquema anti-retroviral de acordo com a gradação do risco do acidente; e

- solicitar sorologia para HIV do paciente-fonte e, caso este seja negativo, suspender a quimioprofilaxia.

b) deve-se solicitar sorologia para HIV, imediatamente após o acidente, para todo indivíduo que sofra exposição ocupacional ao HIV. Caso o resultado do teste seja positivo, encaminhar para acompanhamento específico.

olhos ou boca?; você conhece os procedimentos que devem ser tomados em caso de exposição a sangue contaminado?".

Amostra. O questionário foi preenchido por 136 estudantes do ciclo profissional (terceiro ao sexto ano) do curso de medicina da Faculdade de Medicina da UFMG, antes do início do Curso de Atualização em HIV/AIDS, realizado em novembro de 1996. Esta amostra representa cerca de $10,6 \%$ do total de estudantes deste ciclo.

Análise. Na formação do banco de dados o pacote estatístico Epi-Info 6.02 e para análise o
MULTLR. Após codificados e conferidos, os questionários foram digitados em dois bancos de dados diferentes e comparados posteriormente, para eliminação de erros. Foi realizada análise descritiva de todas as variáveis. As análises univariada e multivariada considerou como variável independente a realização de procedimentos cirúrgicos. As variáveis com $p \leq 0,25$ foram incluídas na análise multivariada. Posteriormente, realizou-se o processo de análise tendo-se como variável independente a exposição prévia a sangue (acidente pérfurocortante e exposição de mucosa ocular e/ou oral). 


\section{RESULTADOS}

A média de idade da população avaliada foi de $22,9( \pm 2,7)$ anos, sendo $66(48,5 \%)$ homens e $70(51,5 \%)$ mulheres. A amostra estudada continha alunos de todos os períodos do ciclo profissional, sendo que $81(59,6 \%)$ estavam no quarto ano do curso de Medicina (Figura 1).

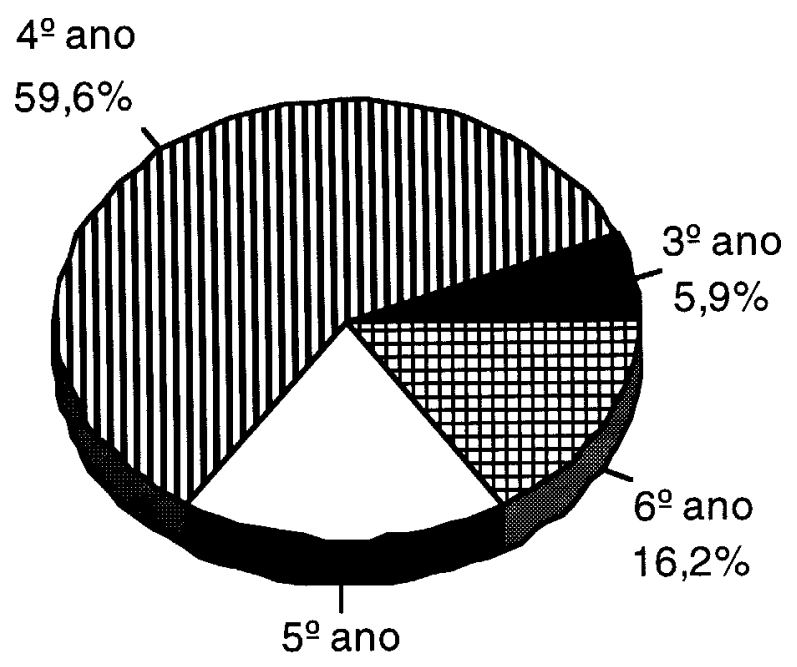

$18,4 \%$

Figura 1 - Distribuição dos alunos de acordo com o ano do curso médico.

A maioria dos entrevistados, 87 (64\%) relatou ter participado de procedimentos cirúrgicos e $68(50 \%)$ relataram já ter sofrido algum tipo de exposição a sangue: $55(44,4 \%)$ acidentes pérfuro-cortantes e $38(27,9 \%)$ exposição de mucosa. Todos os alunos se consideraram em risco ocupacional para infecção pelo HIV ou VHB.

Em relação ao conhecimento das MUB, 90 $(66,2 \%)$ afirmaram conhecê-las, mas apenas 31 $(22,8 \%)$ lembraram-se de alguma matéria do curso médico que as ensinou formalmente. Apenas $33(24,3 \%)$ relataram conhecer os procedimentos a serem tomados em caso de exposição a sangue contaminado.

Setenta e quatro $(54,4 \%)$ estudantes relataram fazer uso das MUB quando especificamente perguntados. Quando questionados sobre medidas específicas, $28(20,6 \%)$ relataram fazer uso de óculos protetor; $97(71,3 \%)$ uso de máscara cirúrgica; 127 (93,4\%) uso de luvas e
$82(60,3 \%)$ uso de avental. Somando-se as quatro variáveis anteriores, tem-se que 128 $(94,1 \%)$ da população faz uso de pelo menos uma das medidas de biossegurança. A maioria dos entrevistados, 105 (77,2\%), afirmaram que o equipamento de biossegurança não lhes pertencia.

A grande maioria da população, 133 (97,8\%), estudada é vacinada contra hepatite $B$.

As Tabelas 2 e 3 mostram os resultados do cruzamento das perguntas realizadas e duas variáveis independentes: realização de procedimentos cirúrgicos e exposição anterior a sangue, respectivamente.

As Tabelas 4 e 5 ilustram os valores de $p$ e as odds relativas observados na análise univariada. $\mathrm{Na}$ análise multivariada, apenas o ano de curso mostrou-se estatisticamente significativo nas duas análises, demonstrando que a realização de procedimentos diminui e que o risco de exposição aumenta com o passar dos anos do curso médico. 
Tabela 2 - Questionário sobre conhecimento teórico e utilização das medidas universais de biossegurança aplicado em 136 estudantes de Medicina. Análise das respostas comparadas com realização de procedimentos cirúrgicos.

\begin{tabular}{|c|c|c|c|c|c|c|c|}
\hline \multirow[b]{3}{*}{ Perguntas } & & \multicolumn{6}{|c|}{ Realização de procedimentos cirúrgicos } \\
\hline & & \multicolumn{2}{|c|}{$\operatorname{sim}$} & \multicolumn{2}{|c|}{ não } & \multicolumn{2}{|c|}{ total } \\
\hline & & $\mathrm{n}$ & $\%$ & $\mathrm{n}$ & $\%$ & $\mathrm{n}$ & $\%$ \\
\hline \multirow[t]{2}{*}{ Sexo } & Masculino & 41 & 30,1 & 25 & 18,4 & 66 & 48,5 \\
\hline & Feminino & 46 & 33,8 & 24 & 17,7 & 70 & 51,5 \\
\hline \multirow[t]{4}{*}{ Ano do curso } & Terceiro & 1 & 0,7 & 7 & 5,1 & 8 & 5,8 \\
\hline & Quarto & 64 & 47,1 & 17 & 12,5 & 81 & 59,6 \\
\hline & Quinto & 16 & 11,8 & 9 & 6,6 & 25 & 18,4 \\
\hline & Sexto & 6 & 4,4 & 16 & 11,8 & 22 & 16,2 \\
\hline \multirow{3}{*}{ Usa óculos? } & Sim & 19 & 14,0 & 9 & 6,6 & 28 & 20,6 \\
\hline & Não & 68 & 50,0 & 39 & 28,7 & 107 & 78,7 \\
\hline & NR & & & & & 1 & 0,7 \\
\hline \multirow{3}{*}{ Usa máscara? } & Sim & 71 & 52,2 & 26 & 19,1 & 97 & 71,3 \\
\hline & Não & 15 & 11,0 & 22 & 16,2 & 37 & 27,2 \\
\hline & NR & & & & & 2 & 1,5 \\
\hline \multirow[t]{2}{*}{ Usa luvas? } & Sim & 87 & 64,0 & 40 & 29,4 & 127 & 93,4 \\
\hline & Não & 0 & 0,0 & 9 & 6,6 & 9 & 6,6 \\
\hline \multirow[t]{3}{*}{ Usa avental? } & Sim & 52 & 38,2 & 30 & 22,1 & 82 & 60,3 \\
\hline & Não & 34 & 25,0 & 19 & 14,0 & 53 & 39,0 \\
\hline & NR & & & & & 1 & 0,7 \\
\hline \multirow[t]{3}{*}{ O material de proteção é seu? } & Sim & 64 & 47,2 & 41 & 30,1 & 105 & 77,3 \\
\hline & Não & 23 & 16,9 & 7 & 5,1 & 30 & 22,0 \\
\hline & NR & & & & & 1 & 0,7 \\
\hline Conhece as medidas universais de & Sim & 56 & 41,2 & 34 & 25,0 & 90 & 66,2 \\
\hline biossegurança? & Não & 31 & 22,8 & 15 & 11,0 & 46 & 33,8 \\
\hline Faz uso da medidas universais de & Sim & 42 & 30,9 & 32 & 23,5 & 74 & 54,4 \\
\hline \multirow[t]{2}{*}{ biossegurança? } & Não & 44 & 32,4 & 17 & 12,5 & 61 & 44,9 \\
\hline & NR & & & & & 1 & 0,7 \\
\hline Lembra de matéria que ensinou & Sim & 18 & 13,2 & 13 & 9,6 & 31 & 22,8 \\
\hline biossegurança? & Não & 69 & 50,7 & 36 & 26,5 & 105 & 77,2 \\
\hline Sabe procedimento em caso de & Sim & 18 & 3,2 & 15 & 11,0 & 33 & 24,3 \\
\hline exposição a sangue contaminado? & Não & 69 & 50,7 & 34 & 25,0 & 103 & 75,7 \\
\hline
\end{tabular}

NR - não relatado

Tabela 3 - Questionário sobre conhecimento teórico e utilização das medidas universais de biossegurança aplicado em 136 estudantes de Medicina. Análise das respostas comparadas com exposição prévia a sangue.

\begin{tabular}{|c|c|c|c|c|c|c|c|}
\hline \multirow[b]{3}{*}{ Perguntas } & & \multicolumn{6}{|c|}{ Exposição prévia a sangue } \\
\hline & & \multicolumn{2}{|c|}{$\operatorname{sim}$} & \multicolumn{2}{|c|}{ não } & \multicolumn{2}{|c|}{ total } \\
\hline & & $\mathrm{n}$ & $\%$ & $\mathrm{n}$ & $\%$ & $\mathrm{n}$ & $\%$ \\
\hline \multirow[t]{3}{*}{ Sexo } & Masculino & 34 & 25,0 & 32 & 23,5 & 66 & 48,5 \\
\hline & Feminino & 34 & 25,0 & 33 & 24,3 & 67 & 49,3 \\
\hline & NR & & & & & 3 & 2,2 \\
\hline \multirow[t]{5}{*}{ Ano do curso } & Terceiro & 0 & 0,0 & 7 & 5,1 & 7 & 5,1 \\
\hline & Quarto & 35 & 25,7 & 44 & 32,4 & 79 & 58,1 \\
\hline & Quinto & 14 & 10,3 & 11 & 8,1 & 25 & 18,4 \\
\hline & Sexto & 19 & 14,0 & 3 & 2,2 & 22 & 16,2 \\
\hline & NR & & & & & 3 & 2,2 \\
\hline \multirow[t]{3}{*}{ Usa óculos? } & Sim & 19 & 14,0 & 7 & 5,1 & 26 & 19,1 \\
\hline & Não & 49 & 36,0 & 58 & 42,6 & 107 & 78,7 \\
\hline & NR & & & & & 3 & 2,2 \\
\hline \multirow[t]{3}{*}{ Usa máscara? } & Sim & 53 & 39,0 & 42 & 30,9 & 95 & 69,9 \\
\hline & Não & 15 & 11,0 & 22 & 16,2 & 37 & 27,2 \\
\hline & NR & & & & & 4 & 2,9 \\
\hline \multirow[t]{3}{*}{ Usa luvas? } & Sim & 64 & 47,1 & 60 & 44,1 & 124 & 91,2 \\
\hline & Não & 4 & 2,9 & 5 & 3,7 & 9 & 6,6 \\
\hline & & & & & & 3 & 2,2 \\
\hline \multirow[t]{3}{*}{ Usa avental? } & Sim & 44 & 32,4 & 35 & 25,7 & 79 & 58,1 \\
\hline & Não & 24 & 17,6 & 29 & 21,3 & 53 & 39,0 \\
\hline & NR & & & & & 4 & 2,9 \\
\hline \multirow[t]{3}{*}{ O material de proteção é seu? } & Sim & 15 & 11,0 & 14 & 10,3 & 29 & 21,3 \\
\hline & Não & 53 & 39,0 & 50 & 36,8 & 103 & 75,8 \\
\hline & NR & & & & & 4 & 2,9 \\
\hline Conhece as medidas universais de & Sim & 50 & 36,8 & 37 & 27,2 & 87 & 64,0 \\
\hline \multirow[t]{2}{*}{ biossegurança? } & Não & 18 & 13,2 & 28 & 20,6 & 46 & 33,8 \\
\hline & NR & & & & & 3 & 2,2 \\
\hline Faz uso da medidas universais de & Sim & 43 & 31,6 & 28 & 20,6 & 71 & 52,2 \\
\hline \multirow[t]{2}{*}{ biossegurança? } & Não & 25 & 18,4 & 36 & 26,5 & 61 & 44,9 \\
\hline & NR & & & & & 4 & 2,9 \\
\hline Lembra de matéria que ensinou & Sim & 18 & 13,2 & 12 & 8,8 & 30 & 22,0 \\
\hline \multirow[t]{2}{*}{ biossegurança? } & Não & 50 & 36,8 & 53 & 39,0 & 103 & 75,8 \\
\hline & NR & & & & & 3 & 2,2 \\
\hline Sabe procedimento em caso de & Sim & 20 & 14,7 & 13 & 9,6 & 33 & 24,3 \\
\hline \multirow[t]{2}{*}{ exposição a sangue contaminado? } & Não & 48 & 35,3 & 52 & 38,2 & 100 & 73,5 \\
\hline & & & & & & 3 & 2,2 \\
\hline
\end{tabular}


Tabela 4 - Análise univariada tendo como variável independente a realização de procedimentos cirúrgicos.

\begin{tabular}{lccc}
\hline Perguntas & $\mathrm{P}$ & OR & IC 95\% \\
\hline Idade & 0,28 & 0,93 & $0,80-1,07$ \\
Sexo & 0,66 & 1,17 & $0,58-2,36$ \\
Ano do curso & $0,01^{*}$ & 0,57 & $0,37-0,88$ \\
Usa óculos? & 0,67 & 1,21 & $0,50-2,93$ \\
Usa máscara? & $<0,01^{*}$ & 4,01 & $1,81-8,87$ \\
Usa luvas?** & - & - & - \\
Usa avental? & 0,93 & 0,97 & $0,47-1,99$ \\
Material de proteção é seu? & 0,12 & 2,11 & $0,83-5,35$ \\
Conhece as medidas universais de biossegurança? & 0,55 & 0,80 & $0,38-1,69$ \\
Faz uso da medidas universais de biossegurança? & 0,07 & 0,51 & $0,25-1,05$ \\
Lembra de matéria que ensinou biossegurança? & 0,44 & 0,72 & $0,32-1,64$ \\
Sabe procedimento em caso de exposição a sangue contaminado? & 0,20 & 0,59 & $0,27-1,31$ \\
\hline
\end{tabular}

$\mathrm{P}=$ valor de $\mathrm{p}\left(\mathrm{x}^{2}\right) ; \mathrm{OR}=$ odds ralativa; $\mathrm{IC}=$ intervalo de confiança.

* Estatisticamente significativo; ** Não é possível calcular, pois uma cas caselas é igual a zero.

Tabela 5 - Análise univariada tendo como variável independente a exposição prévia a sangue.

\begin{tabular}{lccc}
\hline Perguntas & $\mathrm{P}$ & OR & IC 95\% \\
\hline Ildade & $<0,01^{*}$ & 1,67 & $1,28-2,19$ \\
Sexo & 0,80 & 1,09 & $0,55-2,16$ \\
Ano do curso & $<0,01^{*}$ & 2,62 & $1,59-4,32$ \\
Usa óculos? & $0,04^{*}$ & 2,57 & $1,03-6,41$ \\
Usa máscara? & 0,15 & 1,77 & $0,82-3,83$ \\
Usa luvas? & 0,68 & 1,33 & $0,34-5,20$ \\
Usa avental? & 0,50 & 1,27 & $0,63-2,55$ \\
Material de proteção é seu? & 0,91 & 1,05 & $0,46-2,40$ \\
Conhece as medidas universais de biossegurança? & 0,10 & 1,83 & $0,89-3,78$ \\
Faz uso da medidas universais de biossegurança? & 0,17 & 1,62 & $0,82-3,24$ \\
Lembra de matéria que ensinou biossegurança? & 0,27 & 1,59 & $0,67-3,63$ \\
Sabe procedimento em caso de exposição a sangue contaminado? & 0,96 & 1,02 & $0,47-2,24$ \\
\hline
\end{tabular}

$\mathrm{P}=$ valor de $\mathrm{p}\left(\mathrm{x}^{2}\right) ; \mathrm{OR}=$ odds ralativa; $\mathrm{IC}=$ intervalo de confiança.

* Estatisticamente significativo.

\section{DISCUSSÃO}

Os estudantes de medicina da Faculdade de Medicina da UFMG apresentam alto risco de exposição a sangue, que implica em alto risco potencial de exposição ao HIV. A maioria da população, $108(81,2 \%)$, participa de procedimentos cirúrgicos ou já sofreu algum tipo de exposição a sangue (acidente pérfuro-cortante ou exposição de mucosas).

O nível de conhecimento relatado é relativamente baixo; 90 (66,2\%) afirmam conhecer as medidas universais de biossegurança e apenas $31(22,8 \%)$ lembram-se de ensino formal sobre a matéria. Apesar disso, o uso de luvas e máscara é alto, 93,4\% e 72,4\%, respectivamente. No entanto, o uso de óculos de proteção é de apenas $20,7 \%$ e de avental $60,7 \%$.

Considerando o alto risco de exposição ao HIV da população, o baixo nível de conhecimento das medidas universais de biossegurança é preocupante. A discrepância entre baixo nível de conhecimento relatado $(75,7 \%$ não sabem o procedimento em caso de exposição a sangue) e bom nível de utilização de luvas e máscara pode mostrar que a boa prática deve estar relacionada com a rotina de utilização dos equipamentos ou "hábitos de higiene", e não com o nível de conhecimento. A diferença entre o uso de máscaras e de óculos, $72,4 \%$ e $20,7 \%$, respectivamente, parece confirmar esta hipótese, já que praticamente todas as situações que exigem uso de máscara também exigem uso de óculos.

A maioria dos entrevistados relatou que o equipamento de proteção individual (EPI) não Ihes pertencia. Vários estudos, não só na área de saúde, mostram que o aumento da utilização de EPI's deve principalmente a educação e 
supervisão e não a disponibilidade do equipamento. A disponibilização dos EPl's é responsabilidade da instituição empregadora ou de ensino, de acordo com a legislação vigente.

Tanto a realização de procedimentos cirúrgicos e a exposição prévia a material biológico não alteram significativamente o conhecimento e a prática de medidas de biossegurança. Os estudantes que sofreram acidentes não buscaram conhecimento, ou não tiveram acesso a eles. $O$ que realça a atitude negligente dos profissionais de saúde e/ou a falsa sensação de segurança.
A amostra não é aleatória, podendo existir aceitação e desejo de promover assistência a pacientes com AIDS, o que seria sem dúvida outro objetivo a ser atingido, considerando as proporções da epidemia do HIV5.

Deve receber atenção especial a rotina para profilaxia em caso de acidentes. A implantação de serviço de biossegurança, para realização de treinamentos e supervisão em serviço, além do atendimento a acidentes com materiais biológicos, pode auxiliar na modificação do quadro atual.

\section{REFERÊNCIAS BIBLIOGRÁFICAS}

1. Bell D. Occupational risk of human immunodeficiency virus infection in healthcare workers: an overview. American Journal of Medicine 102:9-15, 1997.

2. Blattner W. HIV epidemiology: past, present, and future. FASEB Journal 5:2340-2348, 1991.

3. Centers for Disease Control and Prevention. HIV/Aids surveillance report 1997. Morbidity and Mortality Weekly Report 9:15, 1997.

4. Centers for Disease Control and Prevention. Public health service guidelines for the management of health-care work exposures to HIV and recommendations for postexposure prophylasis. Morbidity and Mortality Weekly Report 47:1-33, 1998.

5. Diekema D, Schuldt S, Albanese M, Doebbeling B. Universal precautions training of preclinical students: impact on knowledge, attitudes, and compliance. Preventive Medicine 24:580-585, 1995.

6. Gottlieb M, Schroff R, Schanker H, Weisman JD, Fan PT, Wolf RA, Saxon A. Pneumocystis carinii pneumonia and mucosal candidiasis in previously healthy homosexual men: evidence of a new acquired cellular immunodeficiency. New England Journal of Medicine 305:1425, 1981.

7. Jones D. Percutaneous exposure of medical students to HIV. Journal of the American Medical Association 264:1188, 1190, 1990.

8. Masur H, Michelis M, Greene J, Onorato I, Stouwe RA, Holzman RS, Wormser G, Brettman L, Lange M, Murray $\mathrm{HW}$, Cunninghma-Rundles S. An outbreak of communityacquired Pneumocystis carinii pneumonia: initial manifestation of celular immune dysfuction. New England Journal of Medicine 305:1431, 1981.

9. Ministério da Saúde. AIDS. Boletim Epidemiológico 11, 1998.

10. Ministério da Saúde. Secretaria de Assistência a Saúde. Programa Nacional de DST/Aids. Documento de consenso sobre a terapia anti-retroviral em adultos. Ministério da Saúde, Brasília, 1997.

11. O'Neill T, Abbot A, Radecki S. Risk of needsticks and occupational exposures among residents and medical students. Archives of Internal Medicine 152:1451-1456, 1992.

12. Resnic F, Noerdlinger M. Occupational exposure among medical students and house staff at the New York City Medical Center. Archives of Internal Medicine 155:75-80, 1995.

13. Siegal F, Lopez C, Hammer G, Brown AE, Kornfeld SJ, Gold J, Hassett J, Hirschman SZ, Cunningham-Rundles C, Adelsberg BR. Severe acquired immunodeficiency in male homosexuals, manifested chronic perianal ulcerative herpes simplex lesions. New England Journal of Medicine 305:1439, 1981. 